Musées, Patrimoine et Culture scientifiques et techniques

142 | 2012

juillet-août 2012

\title{
Les musées, acteurs sur le Web
}

Valérie Schafer, Benjamin Thierry et Noémie Couillard

URL : http://journals.openedition.org/ocim/1077

DOI : 10.4000/ocim.1077

ISSN : 2108-646X

Éditeur

OCIM

Édition imprimée

Date de publication : 1 juillet 2012

Pagination : 5-14

ISSN : 0994-1908

Référence électronique

Valérie Schafer, Benjamin Thierry et Noémie Couillard, «Les musées, acteurs sur le Web », La Lettre de I'OCIM [En ligne], 142 | 2012, mis en ligne le 01 juillet 2014, consulté le 01 mai 2019. URL : http:// journals.openedition.org/ocim/1077; DOI : 10.4000/ocim.1077 
mise en valeur de leurs collections ou la pédagogie à adopter. Cependant, les musées français ont rapidement occupé l'espace du Web, alors même que le grand public est encore peu sensibilisé à l'usage de cette technologie, et ce malgré des réticences initiales, liées autant aux questions propres à l'institution muséale qu'à des problèmes plus généraux, notamment techniques (temps d'affichage des images, lenteur des débits...). La conquête progressive de l'espace virtuel ne se mesure toutefois pas seulement au rythme d'apparition sur la Toile : avoir un site ne signifie pas avoir effectué une réelle acculturation aux usages numériques et il peut y avoir un décalage entre les attentes initiales d'interactivité et des effets plus lents à se concrétiser, à générer des savoir-faire.

À partir de sources issues des archives nationales, des musées (rapports d'activités, colloques...), d'entretiens avec des acteurs du Web (1) dans l'institution muséale, des archives d'anciens sites muséaux, disponibles sur Internet Archive, cet article entend proposer quelques jalons éclairant la manière dont l'institution muséale négocie le virage numérique, en plusieurs temps et à des rythmes différents selon les institutions, tout en essayant toujours de maintenir une image et une voie institutionnelles, elles-mêmes non figées.

Des initiatives isolées à la mise en place de services dédiés et à la généralisation de stratégies de communication sur le Web plus ou moins bien intégrées dans les stratégies (multimédia et globales) des organisations, d'un fonctionnement vertical à l'expérimentation d'un «modèle 2.0 » plus horizontal et collaboratif, se dessine autant la relation des musées français au numérique, quà ses missions, ses publics, son organisation.

\section{Voies rapides et chemins de traverse}

La rencontre du musée avec le numérique ne débute pas avec le Web. Le Minitel a aussi connu des expériences, à l'image du « 3614 Joconde » qui donne accès à une base de données textuelle de 120000 descriptions de peintures, dessins et gravures de 60 musées français. Toutefois le Web introduit une rupture que certains musées vont saisir rapidement, à l'image du musée des Arts et Métiers, dès 1994. Plus encore, la décennie 2000, qui connaît une diffusion très rapide de linformatique personnelle, est celle du développement des possibilités d'une communication à la fois de masse et personnalisable. De nouvelles opportunités émergent, auxquelles les musées vont s'adapter, avec plus ou moins de spontanéité et de savoir-faire.

\section{Poids lourds, conduite accompagnée} ou sans permis

Sans surprise, les premiers musées qui s'installent sur le Web sont d'abord ceux qui ont précocement investi le multimédia et qui en ont les moyens. En 1994, le site du ministère de la Culture montre l'exemple. La même année une exposition virtuelle sur L'âge des Lumières, montée en collaboration avec l'INRIA, réunit 18 musées. Le musée des Arts et Métiers se lance aussi dans l'expérience et devient un précurseur, avec la mise en ligne du catalogue des collections. Il va plus loin en partageant son savoirfaire et son espace, permettant à des partenaires de mettre à disposition des bases de données à partir de son site, pour peu qu'elles soient d'intérêt public. Ses capacités, rares pour l'époque, sont mises au service de l'édition en ligne, par exemple pour l'Écho des régions et $\mathrm{PenWeb}$, tandis que la mise en valeur

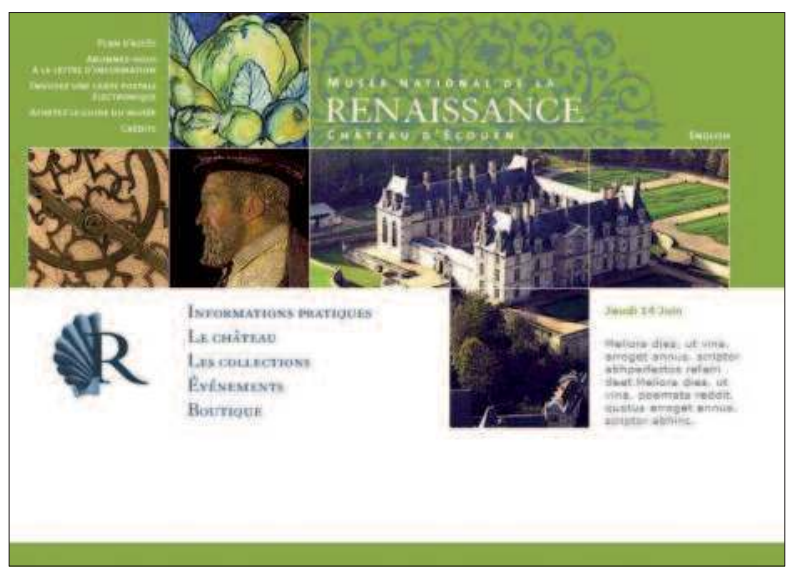

Page d'accueil du site Internet du musée de la Renaissance, avant 2007 (sur le modèle Réunion des Musées Nationaux)

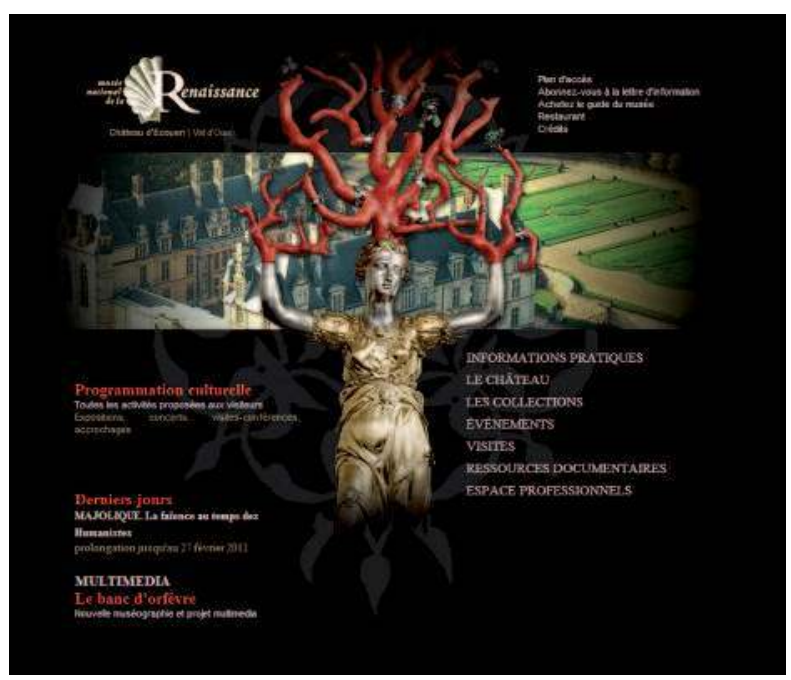

Page d'accueil du site Internet du musée de la Renaissance, après 2007 (avec l'aimable autorisation du musée national de la Renaissance - château d'Écouen). 
pédagogique du pendule de Foucault et d'autres pièces remarquables ouvre une voie pionnière (ElardjaProuzeau, 1999). Le site dépasse déjà la simple vitrine. La dimension pédagogique est pleinement intégrée, à l'image des débuts sur le Web de bien des musées des sciences et techniques ensuite.

Parallèlement des initiatives dispersées, parfois nées hors les murs de l'institution, apparaissent. Une des plus originales est dès 1994 le site indépendant « Weblouvre ", développé par un jeune polytechnicien, qui pousse le musée à réagir. Les circonstances mêmes de sa mise en ligne en font un produit inattendu dans la politique culturelle du Louvre : "Le nom de domaine du Louvre est repris à un étudiant d'une école d'ingénieur, 'cybersquatter' qui s'en est emparé pour en faire un site personnel. La récupération du nom conduit naturellement à la création d’un site »(Prot, 2003). La loi du premier arrivé et l'anarchie propre au premier temps du « cyberespace » ne s'appliquent que peu de temps.

Les initiatives émanent aussi de quelques conservateurs. Philippe Avenier, à la Direction des Musées de France, se souvient : "Le conservateur du musée des Beaux-Arts de Bordeaux a souhaité créer son site dès 1995. Mais à l'époque, personne ne voulait miser sur l'Internet et les sociétés de conception de pages Web n'existaient pas. Nous avons donc fait un travail d'artisan, en échangeant nos trucs et astuces avec d'autres collègues » (2). Malgré les quelques enthousiastes qui développent des sites de manière artisanale (au musée des Arts Décoratifs, le premier animateur du site est le gardien de nuit qui s'intéresse à cette technologie (3)), il faut noter l'absence de personnels réellement qualifiés.

Si quelques musées tentent l'expérience de manière spontanée, d'autres vont bénéficier du soutien de la Direction des Musées de France et de la Réunion des Musées Nationaux, qui proposent un programme de mise en réseau, dont ils soutiennent la réalisation. Les actions s'inscrivent aussi à partir de 1998 dans le cadre du Plan d'action gouvernemental pour la société de l'information, qui fait du domaine culturel un axe prioritaire pour le développement du "réseau des réseaux » et de ses usages. Ceux-ci sont notamment pensés comme support pédagogique et, en février 2000, est prise l'initiative d'une «boucle des contenus » à haut débit. Celle-ci s'appuie sur le constat que si la France a atteint 5 millions d'internautes et si les équipements dans les établissements d'enseignement n'ont cessé de croître, le nombre de serveurs est insuffisant et les internautes tentés de chercher leurs informations à létranger en raison de la faiblesse des débits d'accès des sites français. La « boucle des contenus » relie à partir de février 2001 Renater (réseau national des télécommunications pour la Technologie, la Recherche et l'Enseignement) et différents sites (BnF, ministère de la Culture, Cité des Sciences et de l'Industrie...) par des liaisons à 34 ou $155 \mathrm{Mbits} / \mathrm{s}$. Les régions sont aussi sources d'initiatives et, en 1999 par exemple, la Région Poitou-Charentes peut s'enorgueillir de la mise en ligne de pages mettant en avant l'identité des musées de la région.

\section{Professionnalisation et externalisation, opérateurs et manufacturiers}

Les progrès en terme de fréquentation sont spectaculaires sur les sites blockbusters. En 2000, le musée du Louvre compte 6 millions de visites sur son site, soit autant que de visiteurs réels, mais il note une crise de croissance et dès 2001 une seconde version du site est développée, soutenue par les cellules mécénat. Pendant 4 ans une quinzaine de consultants s'activent sur le projet Cim@ise, dont le coût est estimé entre 6 et 8,5 millions d'euros (hors coût des bases de données et salaires des 60 personnes mobilisées). Pour évoluer régulièrement et séduire, les sites réclament une professionnalisation et un investissement humain et financier.

Au cours de ces mêmes années 2000, certains musées souhaitent se dégager du «modèle RMN » pour s'offrir une identité Web plus personnalisée. Le musée Guimet propose en 2006 un nouveau site, dont la charte graphique est repensée en s'appuyant sur des références aux collections : la composition verticale se veut un rappel des kakemonos et du logo du musée, les couleurs choisies sont symboliques en Chine ${ }^{(4)}$. Ce cas n'est pas isolé et il illustre, en plus de l'utilisation d'un outil libre, l'apparition de nouveaux acteurs : les entreprises spécialisées en Web Design et l'investissement des mécènes dans ce champ.

Au sein de l'institution muséale, les responsables de sites commencent à venir de formations ayant intégré le multimédia. L'Institut national du Patrimoine (INP) s'est engagé dès 1996 dans une politique de formation aux nouvelles technologies à destination des conservateurs et des professionnels du patrimoine. En formation initiale, les futurs conservateurs suivent notamment un séminaire pratique de conception d'un site Internet. D'utilisateurs de bases de données qu'ils étaient presque tous en arrivant à l'INP, ils se préparent à devenir acteurs et prescripteurs. L'enseignement des nouveaux outils et du multimédia s'étend à la plupart des grandes écoles d'art. Certaines en font un axe fort de leur formation. L'École 


\section{La Cité nationale de l'Histoire de l'Immigration, du site qui précède le musée à I'investissement dons les résecux socio-numériques}

La version 1 du site Internet de la CNHI date de 2003, avant l'ouverture de la CNHI en 2007 : le site virtuel a précédé le musée et était conçu d'emblée comme complémentaire.

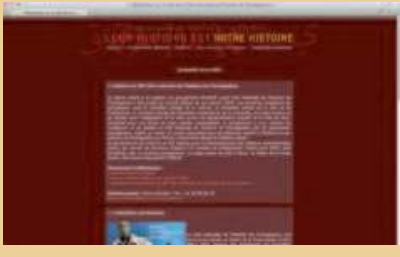

version 1

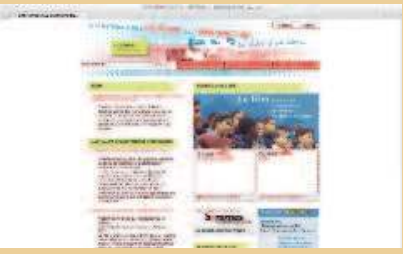

version 2

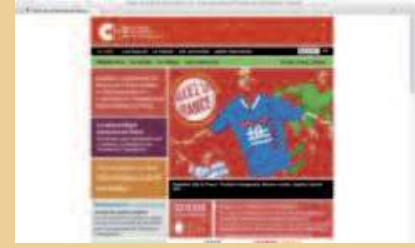

version 3 (à partir de 2006)
Le site actuel : la navigation gagne en complexité et les itinéraires proposés en profondeur au fil des versions. La page s'enrichit de menus latéraux.

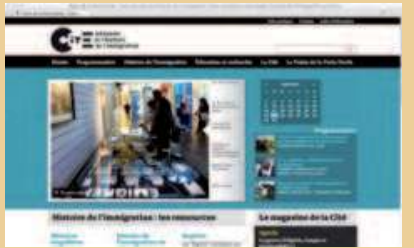

application sans réelle dissociation des informations et services présents sur le site (internet ou réel). L'autonomisation du recours aux dispositifs mobiles se

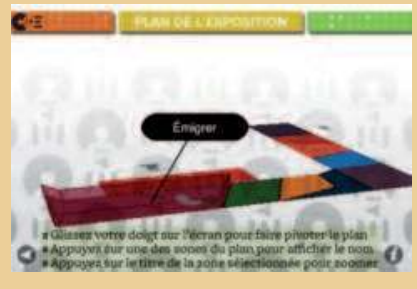
fait attendre... Plan interactif, elle permet néanmoins une adaptation aux souhaits du visiteur dans le cadre d'un itinéraire personnalisable.
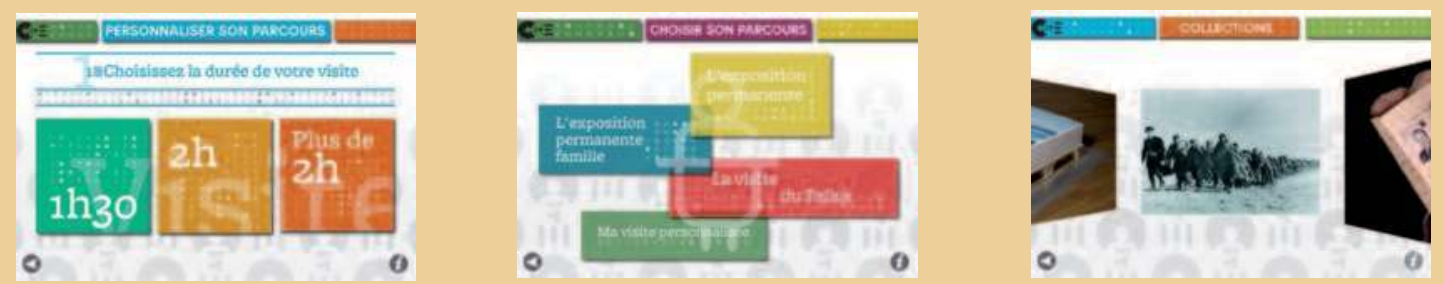

De l'information et de la communication institutionnelle, à la communication partagée ? Sur Twitter et Facebook, les followers et amis représentent des volumes modestes, mais rendent la politique d'information plus participative en attendant d'être véritablement interactive.
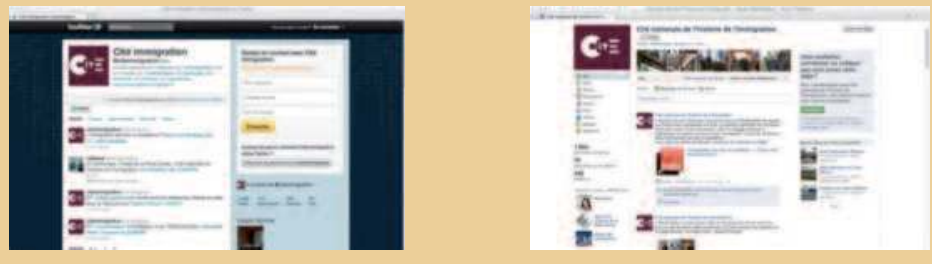
nationale supérieure des Beaux-Arts met en place un mastère spécialisé en multimédia-hypermédia dès 1995. L'École nationale supérieure des Arts décoratifs (ENSBA) délivre deux post-diplômes spécialisés: «Images de synthèse et effets spéciaux » et depuis 1999 un post-diplôme expérimental en commun avec Paris 8, l'atelier de recherches interactives. Certaines écoles créent conjointement avec l'université des troisièmes cycles. C'est le cas notamment de l'ENSBA (avec l'EHESS), de l'École nationale supérieure de la Création industrielle (ENSCI) (avec Paris 8), de l'École supérieure de l'Image de PoitouCharentes ("Arts numériques » avec les universités de Poitiers et La Rochelle), de l'École de Montpellier («Arts et ville» avec l'université de Montpellier 3 et un laboratoire du CNRS ${ }^{(5)}$ ).

Ces formations sont parfois stimulées par le ministère (Sites Internet des musées: nowveaux formats, nouveaux usages, Direction générale des Patrimoines), des conférences sont dédiées à la relation des musées au TIC (Le musée en ligne, le 13 juin 2007, Articuler le musée et les réseaux socianx, le 17 décembre 2008, ou encore Réseaux socianx, hauts débits, nouveaux médias, nowvelles pratiques sociales et pédagogiques, le 23 mars 2010, toutes organisées par l'Institut de Recherche et d'Innovation) tandis que l'Office de Coopération et d'Informations Muséales (OCIM) propose aux professionnels d'échanger sur leurs pratiques.

Il demeure toutefois difficile de parler d'une profession unifiée par des pratiques, une formation et une organisation, tant les responsabilités et les fonctions sont multiples. D'un musée l'autre, les concepteurs et animateurs des sites n'ont pas les mêmes titres et missions. Les équipes sont de taille variable. Elles vont de l'agent unique au sein de l'institution en lien avec les prestataires extérieurs et les autres services du musée (Guimet, Arts Décoratifs) à des équipes plus étoffées et clairement distinguées au sein de l'organisation interne comme au Louvre. Toutefois, des initiatives comme Museomix, au musée des Arts décoratifs en novembre 2011, permettent à une communauté en cours de formation et de structuration d'échanger pratiques et expériences, tout en s'ouvrant à d'autres. Parallèlement, le recours à des prestataires de services devient la règle pour le développement de pages plus riches qu'il serait trop coûteux de produire en interne (OCIM, 2007). Lutilisation de logiciels Open Source (Spip ou TikiWiki pour Guimet, Joomla pour la CNHI (6)), garantit la continuité par l'utilisation de solutions libres et bien documentées, qui permettent d'envisager des coûts plus modestes et un changement de prestataire sans rupture dans la gestion des pages. L'externalisation de la réalisation répond à une demande de savoir-faire et d'expertise et une volonté d'attractivité à l'égard d'internautes de plus en plus sollicités. D'une offre de pénurie à une offre pléthorique, d'un modèle top-down de diffusion des contenus à un renversement, au moins dans les discours, vers des initiatives présentées comme bottom-up, les musées se retrouvent face à des utilisateurs, de plus en plus exigeants, qui passent du statut de débutant dans les années 1990 à celui « d'internautes 2.0 » à partir du milieu des années 2000.

\section{De la verticalité à I'horizontalité ?}

La manière dont les professionnels de la culture négocient le virage numérique ne peut être comprise sans la replacer dans le contexte de l'histoire muséale, mais aussi technique. L'évolution du Web est rapide depuis les années 1995. Dans un univers en réseau qui subit des changements notables, les musées évoluent d'un modèle très hiérarchique, vertical, vers des tentatives d'ouverture qui passent par la communication

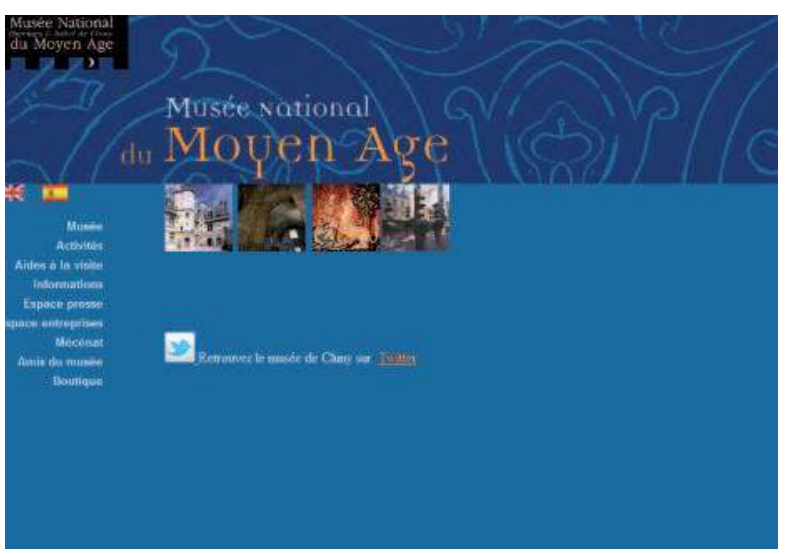

Une page d'accueil sur le modèle Réunion des Musées Nationaux qui perdure au musée national du Moyen-Âge...

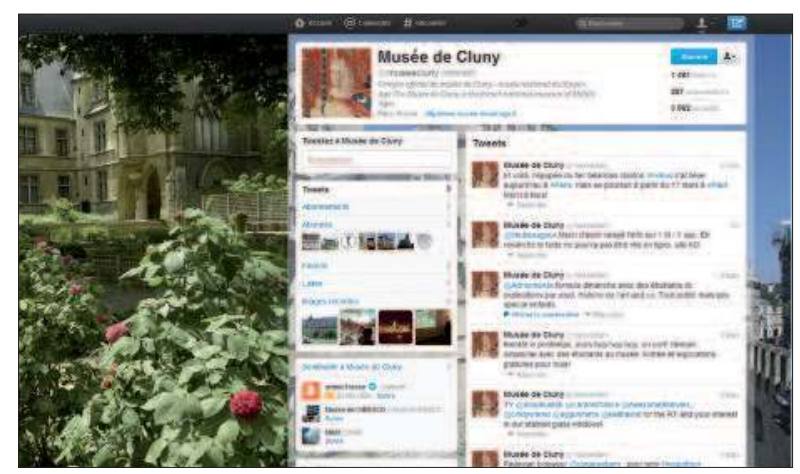

..mais un investissement dans les réseaux socio-numériques avec un dialogue quotidien sur Twitter. (c) RMN-Musée du Moyen Age/Mosquito 
via les réseaux socio-numériques ou des accords avec des acteurs comme Google ou les membres de Wikimedia. Les relations des professionnels au sein du musée avec les autres acteurs de l'institution sont le fruit de négociations constantes, négociations qui s'étendent maintenant aux internautes, en particulier avec des amateurs éclairés, et aux acteurs puissants du Web, pour qui l'art est un marché de contenus à conquérir.

\section{De l'occupation de la Toile au site} multifonctionnel, des craintes de désertion

\section{à la visite augmentée}

L'exploitation d'Internet par les institutions muséales françaises peut être scindée schématiquement en deux phases, sans que l'on puisse toujours saisir clairement la césure entre les deux et sans que les derniers arrivés sur la Toile ne fassent nécessairement l'économie de la première, pour directement bénéficier du savoir-faire de ceux qui se sont engagés plus précocement. La logique suivie est globalement d'abord celle de l'occupation du terrain : il s'agit d'avoir son site, en suivant l'exemple de quelques grandes institutions déjà dotées. Les résultats de l'étude réalisée par l'OCIM en 2007 relèvent que « le seul 'fait d'exister' constitue un de ces points forts : 'ce site [...] a le mérite d'exister, et donc nous existons' ». Les initiatives menées en interne, parfois par un autodidacte, ne permettent pas encore la mise en place d'une véritable politique de communication en ligne, tandis que l'on teste toutes les nouvelles technologies, par exemple Flash dans les années 2000. Au suivisme, qui n'est pas le propre des institutions de musées, s'ajoute une grande diversité des formes. L'impératif techniciste peut l'emporter sur la réflexion de fond. Jusqu'en 2006, le site du musée du Louvre offre une page d'accueil tripartite, avant de développer l'arborescence en profondeur. Cette complexification progressive illustre l'apprentissage collectif des nouvelles technologies, nécessaire à une véritable communication en ligne. Celle-ci n'est pas liée à une époque, mais plutôt à une chronologie propre à chaque institution.

La seconde étape consiste à envisager le site comme un véritable outil au service des publics, à le sortir d'une logique de vitrine pour partir à la recherche de la richesse fonctionnelle, penser la préparation de la visite, proche ou lointaine, pratique ou davantage tournée vers le souci de susciter le désir, de manière dynamique. Parmi les premiers signes d'un souci de renforcer l'aspect pratique mais aussi dans la logique de concevoir la visite et la venue in situ, la RMN lance en 1996 un système de réservation en ligne. La vente de produits en ligne, avec les cyber-boutiques en 1999, répond à une logique différente : les modalités de la commercialisation de la culture commencent à s'exprimer avec de nouveaux supports dans l'espace domestique. En 1999, avec le Louvre.edu, les services s'étoffent encore : l'œuvre elle-même entre au rayon des produits proposés en ligne. Là encore, la démarche est toutefois indissociable d'une
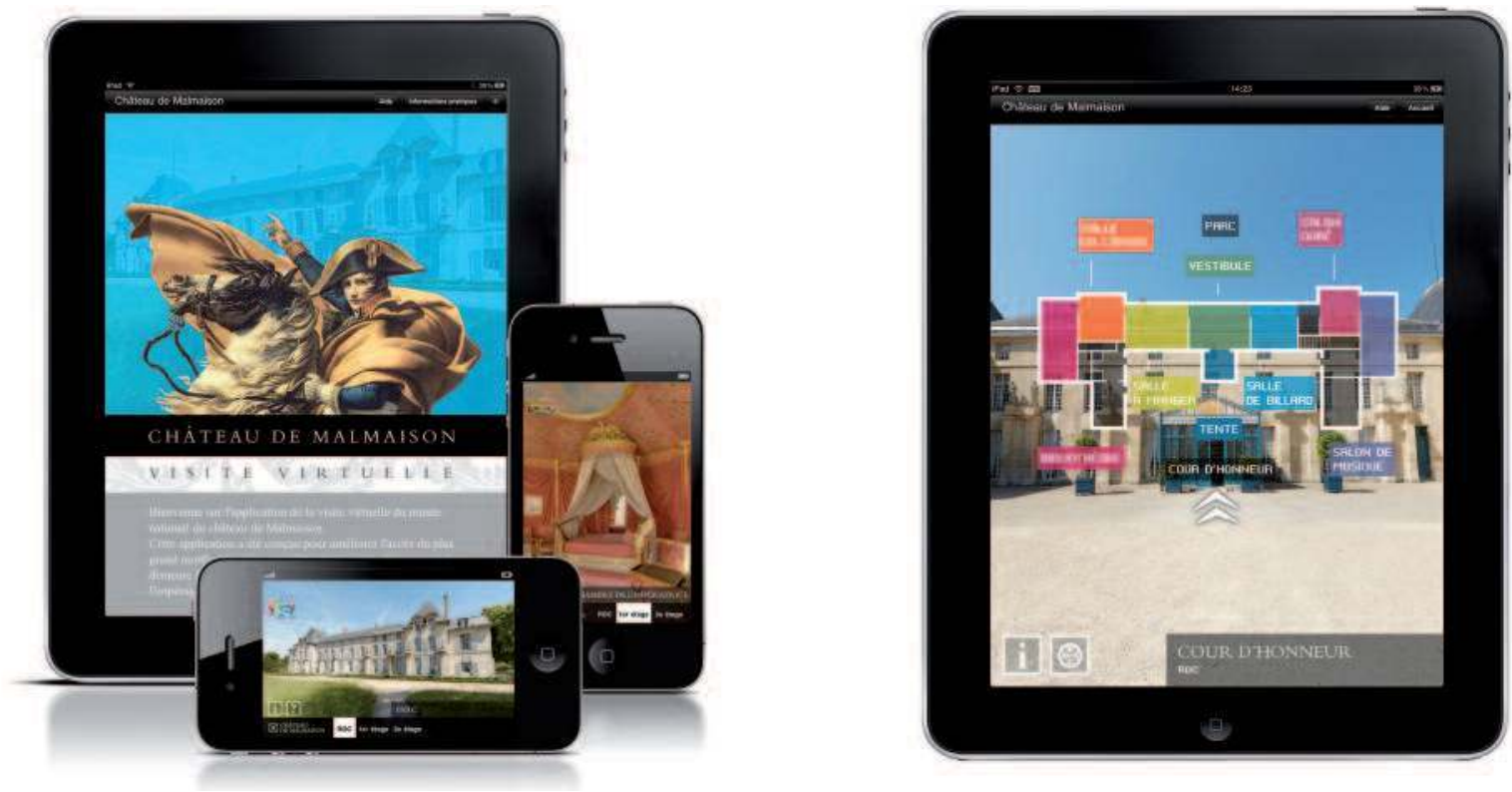

L'application iPhone et iPad du musée national du château de Malmaison. L'application permet une visite virtuelle du château à $360^{\circ}$. Le projet arrive à son terme au premier trimestre 2012 avec la mise en place à l'accueil du musée d'un dispositif de prêt à destination des personnes à mobilité réduite de cinq iPad permettant de pallier l'inaccessibilité de certaines salles. ○) Musée national du château de Malmaison/Mosquito 
volonté de segmenter l'offre pour répondre à l'attente de différents publics et doit être replacée dans le cadre d'une prise de conscience précoce des usages pédagogiques possibles du site. La médiation culturelle et l'adaptation aux publics sont de plus en plus intégrées (espaces pour les enfants, par exemple).

Au sein de cet accroissement des fonctions offertes, une tendance est à la conversion des outils professionnels des musées en ressources de données exploitables par le grand public. Essentiellement architecturée autour de la mise en ligne de bases de données documentaires, cette pratique illustre la volonté des musées d'enrichir leur offre Internet, au moins en terme quantitatif. C'est notamment ce que le musée du quai Branly a réalisé avec la mise en ligne des 300000 fiches descriptives (7). La richesse des contenus reste prioritaire.

La volonté de montrer le musée via des panoramiques, la retransmission de conférences par des musées qui sont aussi des espaces de rencontres, le souci de montrer les hommes et les coulisses de l'institution (8) sont en outre dans quelques grands musées français le résultat de véritables plans de communication qui commencent à être négociés au sein de l'institution. La collection est loin d'être le seul atout des musées, qui peuvent aussi compter sur leur patrimoine architectural, l'identité des lieux pour susciter l'intérêt. Lorganisation se donne aussi à voir : le musée se pense sur le site au-delà du cloisonnement des salles et des collections comme un tout. Toutefois l'élaboration d'une politique collective, négociée, raisonnée, faisant intervenir pleinement les différentes parties prenantes n'est pas encore généralisée et les enjeux de pouvoir internes sont parfois prégnants.

Les sites ont aussi une volonté de narration, de mise en récit et en intelligibilité qui s'apparente à la visite guidée (Ben Sassi, 2007). Celle-ci s'enrichit de la multiplication récente des dispositifs de poche (téléphones portables, tablettes), qui suscitent la création d'applications ou de services destinés à préparer, continuer, mais également « augmenter » l'expérience de la visite. Ainsi, au Grand Palais, il est possible depuis 3 ans de charger des informations à partir de bornes interactives bluetooth. De même, dans le sillage du succès de l'iPhone et de l'iPad, les institutions se lancent dans la production d'applications mobiles, qui se positionnent comme un dépassement de l'audioguide et permettent une « visite augmentée ». Elles proposent, en plus des traditionnelles informations pratiques, d'accéder à des commentaires d'œuvres (notices, vidéo, commentaires audio) et de

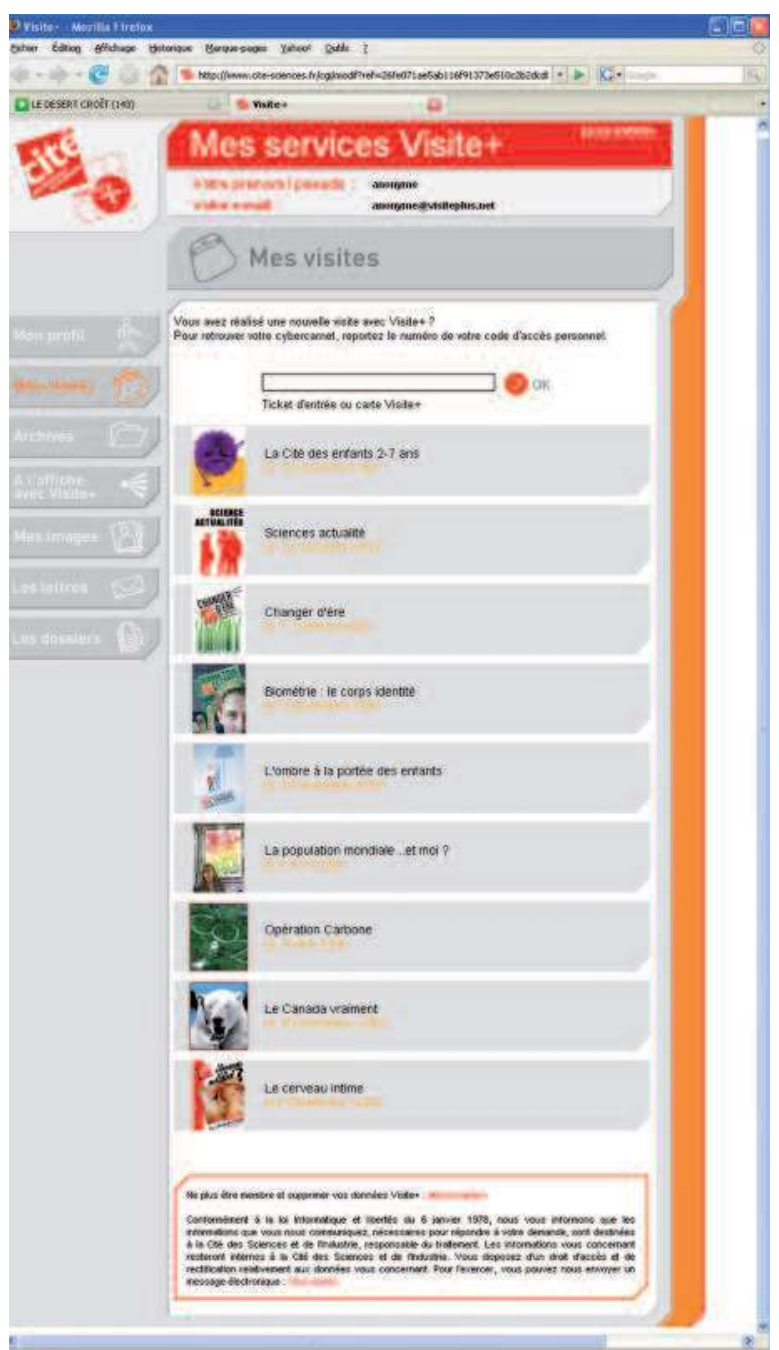

Le portail du visiteur membre de Visite+ avec le rappel de ses visites et l'accès aux autres offres de la Cité des Sciences et de l'Industrie (c) CSI

zoomer sur des détails. Quelques-unes permettent d'accéder à de la réalité augmentée : en visitant les jardins de Versailles des informations apparaissent à des bosquets précis et des personnes en $3 \mathrm{D}$ commentent au musée des Beaux-Arts de Rennes les œuvres regardées à travers son smartphone (Dehon, 2011). La visite sur place est toutefois toujours conçue comme l'élément central d'une visite plus globale qui commence devant un écran pour planifier sa venue, est soutenue in situ par les informations disponibles via son téléphone sur les parcours de visite, et s'achève à nouveau par écran interposé avec la consultation d'informations complémentaires. Cette offre post-visite s'étend même dans le cas de l'exposition L'hôtel particulier. Une ambition parisienne, à la Cité de l'Architecture, à une visite hors-les-murs : les hôtels parisiens sont géolocalisés grâce à une application mobile et 
expliqués par des notices. Des initiatives originales telle que Visite +, de 2002 à 2007 dans les expositions de la Cité des Sciences et de l'Industrie, permettent un moment "post-visite » personnalisé et donnent au musée le moyen de connaître davantage ses visiteurs (Topalian, Le Marec, 2007). Le dernier avatar de ces innovations est l'entrée dans l'ère de la réalité augmentée stricto sensu : expérience au musée du Moyen-Âge de Cluny ou au château de Vincennes en 2009, expérience immersive au Petit Palais à l'automne 2012 avec 3D... (Chapelain, 2011).

Vers le « musée 2.0 »?

Vers 2005, le Web connaît un tournant vers ce que Tim O'Reilly nomme le Web 2.0 ou Web social. Si avec le « Web 1.0 » les contenus émanent de ceux qui ont la maitrise des outils informatiques pour pouvoir publier, la publication de contenus s'est ouverte ${ }^{(9)}$, avec un Web structuré par et autour des utilisateurs (dont il conviendrait toutefois de montrer certaines limites). Ces applications sont rapidement adoptées à des fins de publicité et de communication car le Web social est aussi un instrument du Web marchand et marketing. Les musées se saisissent de plus en plus de ces outils depuis 2008 (notamment de Facebook) pour valoriser leurs actions et leurs collections sur des sites regroupant des millions d'utilisateurs. La présence des musées sur les réseaux socio-numériques s'est faite par vagues en France, bien après les grands musées canadiens et nord-américains. Les pionniers d'une présence multiplateforme sont en 2008 les Abattoirs de Toulouse, le muséum d'Histoire naturelle de Toulouse et la Cité des Sciences et de l'Industrie dont les domaines d'intervention (sciences et art contemporain) ont été très tôt confrontés aux nouvelles technologies (Ben Sassi, 2004). Une deuxième vague touche les musées d'histoire, d'ethnographie, de civilisations et de beaux-arts vers 2010 (Couillard, 2010). Il ne s'agit toutefois pas d'une conversion à une co-construction égalitaire des savoirs, dont les limites ne tiennent pas seulement à une réticence muséale (Chaumier, 2008) mais se heurtent à un principe de réalisme. Les fonctionnalités sont utilisées dans plusieurs directions. D'une part, elles permettent les interactions entre les membres et le musée. Une étude autour de jeux proposés par le musée des Arts décoratifs tend à montrer que ces interactions sont tournées beaucoup plus vers le musée que vers la constitution d'une communauté autour du musée (Couillard, 2010). D'autre part, elles peuvent laisser espérer un élargissement des publics, notamment en touchant la tranche des 25-45 ans et le public étranger. Enfin, elles peuvent aussi s'appuyer sur l'aspect viral de ces nouveaux outils, pour faire de la page Facebook un élément de valorisation du site Internet.

Contournant les critiques à l'encontre du plus célèbre des réseaux sociaux et profitant d'un partenariat d'innovation avec Orange, le Louvre lance fin 2010 sa «Communauté Louvre ». Des aspects techniques et structuraux sont cependant communs aux deux sites : création de profils, de réseaux d'amis, possibilité de former des groupes d'intérêt, de s'envoyer des messages, d'écrire des articles et d'identifier des photographies. Cette plateforme sert d'expérimentation : le Louvre tente le pari d'une structuration des actions proposées au sein de la plateforme par les internautes eux-mêmes, sans l'intervention du musée. Le site a fermé le 18 octobre 2011 et pour l'instant ce projet pilote n'a pas donné lieu à son incorporation au sein du nouveau site Internet, mais cette phase expérimentale témoigne d'une volonté de penser les outils en passant par une phase concrète de pratiques et d'expérimentation sociale. S'inspirant également de la tendance collaborative, le Château de Versailles s'associe à Wikipedia en 2011 pour un projet de six mois : un « wikipedien » est accueilli au château pour faire découvrir l'encyclopédie libre en ligne au personnel, tandis que des passionnés contribuent à la création et à l'enrichissement des notices dédiées au musée sur le site. Le Château de Versailles a également conclu un partenariat avec Google pour son Art Project qui consiste en la numérisation panoramique

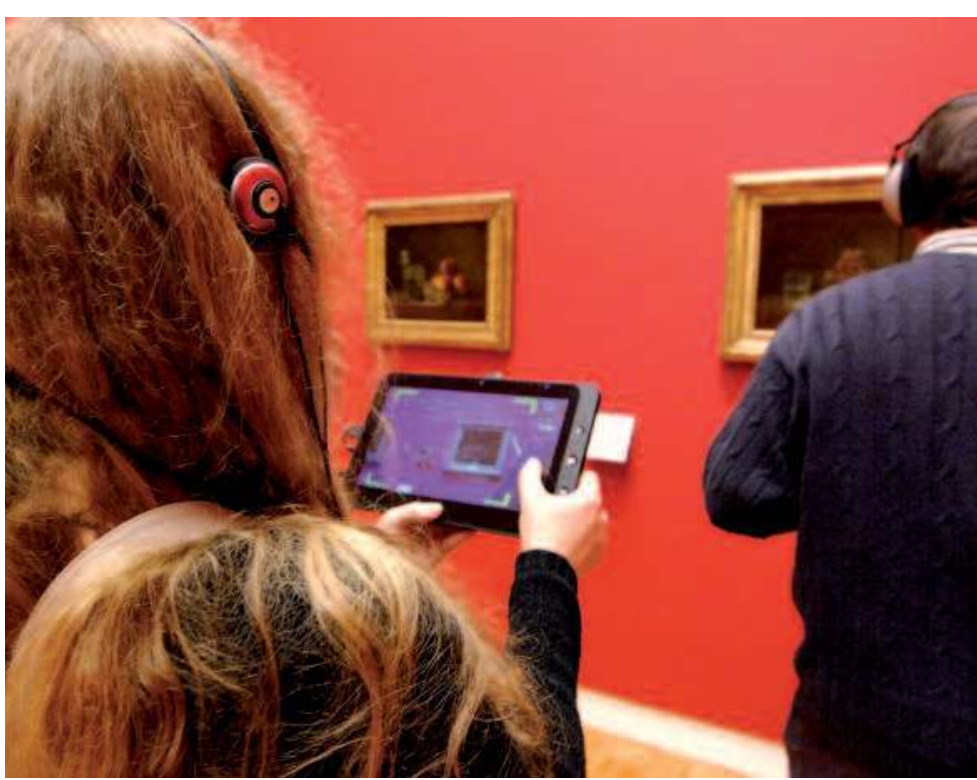

Le musée des Beaux-Arts de Rennes espère séduire le public des 15-25 grâce à son application qui dévoile les secrets des œuvres. (๑) Musée des Beaux-Arts de Rennes/Jean-Manuel Salingue 
de salles de 17 musées dans le monde (avec la technologie Street View utilisée pour Google Maps), ainsi que celle à très haute résolution de plus de mille œuvres. Ces deux projets peuvent sembler contradictoires du point de vue des logiques communautaires, puisque le portail sur Wikipedia promeut la collaboration et la réutilisation des données sous licence Creative Commons, quand Google ne permet pas le téléchargement des œuvres numérisées sous l'argument du droit d'auteur, même pour des œuvres tombées dans le domaine public. Mais, ils sont cohérents dans l'optique d'une valorisation du patrimoine, reposant sur tous les supports des nouvelles technologies de l'information et de la communication : le Château de Versailles fait à la fois le choix de la visibilité et d'une tentative de coconstruction de savoirs. Ainsi, en nouant des partenariats avec les empires du Web, les grandes institutions peuvent espérer renforcer leur place sur l'échiquier des grands musées internationaux et contribuent à légitimer en retour des entreprises de la communication, qui bénéficient ainsi d'une image culturelle et sociale respectable.

L'évolution constante de la présence en ligne des musées depuis 1994, qui tient autant à des évolutions technologiques et aux tendances que connaît le Web même et l'émergence des réseaux socio-numériques, qu'à l'évolution des politiques muséales et des acteurs qui les portent, ne permettent pas de parler de stabilisation d'un modèle, encore assez marqué par le pragmatisme et l'expérimentation. Si les logiques d'acteurs se précisent, si la vocation du site répond aussi à la mission historique de l'institution qui « caractérise des rapports socianx spécifiques et le recours à la communication de la part des musées »(Vidal, 2003), le site du musée est autant le reflet d'une organisation interne que de sa relation aux publics.

Le musée a réussi à négocier le virage de l'interactivité relevant de la communication homme-machine, qui rejoint les problématiques générales d'accès à l'information au moyen de l'ordinateur. L'interactivité sociale, c'est-à-dire la mise en place d'un dialogue avec l'utilisateur du site ou même la construction d'un «visiteur-acteur » du musée en ligne, est en cours de négociation, car le virage numérique ne peut plus s'emparer des nouveaux outils sans penser aussi les usages et usagers.

\section{Notes}

(1) La campagne d'entretiens et le dépouillement des archives ont notamment été menés en 2010-2011, par Noémie Couillard dans le cadre de son Master 2 de recherche en muséologie (Présence muséale dans le «Web social » : enjeux, pratiques et effets), et par Benjamin Thierry et Valérie Schafer dans le cadre du colloque international « Nouveaux musées, nouvelles ères urbaines, nouvelles mobilités touristiques », co-responsables scientifiques Édith Fagnoni et Maria Gravari-Barbas, Paris : Société de Géographie, 20 et 21 janvier 2011.

(2) Moreau, D. Les musées nationaux virtuels sont surtout visités par les Américains : chefs-d'œuvre à portée de souris, Le Figaro, 10 décembre 1999. Voir également Avenier, P. La numérisation à la Direction des Musées de France, Le Figaro, 19 novembre 1999.

(3) Escalona, F. entretien du 25 novembre 2010, musée des Arts décoratifs.

(4) Mussard M., entretien du 8 décembre 2010, musée Guimet et Schafer, V. et Thierry, B. Le mariage de raison du musée d'art et du Web, Les musées au prisme de la communication, Hermès nº1, 2011, p. 103. (5) CAC Fontainebleau, Carton 20050055, article 3.

(6) Sagot R., entretien du 21 décembre 2010, Cité nationale de l'Histoire de l'Immigration.

(7) Chassagne C., entretien du 6 janvier 2011, musée du quai Branly.

(8) Cette tendance à «personnaliser » l'institution, à rendre les hommes visibles est intéressante : elle est poursuivie notamment via les outils 2.0. Ainsi, comme le remarque Laetitia Aubin : «De même, au muséum de Toulouse, les internautes interagissent non plus avec une image abstraite et impersonnelle de l'institution mais avec des profils, ceux des deux webmasters du musée : Samuel et Aude». Aubin, L. S'approprier la visite au musée : quels modèles pour la participation amateur? Mémoire de Master 2, Histoire et Politique du patrimoine et des musées, université Paris 1 Panthéon-Sorbonne, 2011. http://observatoire-critique.hypotheses.org/files/ 201 1/09/Mémoire_M2_Aubin.pdf

(9) Rieder, B. De la communauté à l'écume : quels concepts de sociabilité pour le « Web social » ?, TicEsociété, vol. 4, n¹. http://ticetsociete.revues.org/822

\section{Bibliographie}

Ben Sassi, M. Le musée à l'ère d'Internet. Mémoire de Master 1, université Paris 1 Panthéon-Sorbonne, 2007.

Chapelain, B. De nouvelles médiations numériques au service de la culture augmentée, Hermès, n61, 2011

Couillard, N. Présence muséale dans le Web social : enjeux, pratiques et effets. Mémoire de recherche, $2^{\mathrm{e}}$ année de $2^{\mathrm{e}}$ cycle, École du Louvre, 2010,167 p.

Dehon, C. Le téléphone portable, nouvel outil de médiation culturelle dans les institutions muséales françaises. Mémoire de master 1, université Paris 3 Sorbonne Nouvelle, 2011. www.scribd.com/doc/62562855/Le-telephone-portable-nouvel-outil-de-mediation-culturelle-dans-les-institutionsmuseales-francaises

Elardja-Prouzeau, M. Le site du Web du musée national des arts et métiers : un site de ressources pour les professeurs de technologie, Revue de l’EPI, n96, 1999. www.epi.asso.fr/fic_pdf/b96p157.pdf 
OCIM Les institutions muséales et leur site Internet. Synthèse et résultats de l'étude réalisée par l'OCIM, septembre-novembre 2007.

Mairesse, F. Les musées et Internet. Les enjeux de la numérisation, in Association des Cercles francophones d'Histoire et d'Archéologie de Belgique, 8 e Congrès / Fédération des Cercles d'Archéologie et d'Histoire de Belgique, 55e Congrès, 23-31 août 2008, Namur : Presses universitaires de Namur, t. 4, 2011.

Prot, M. Naissance du projet Cim@ise de refonte du site Internet. École du Louvre, 2003. http://www.archimuse.com/publishing/ichim03/119C.pdf

Schafer, V. et Thierry, B. Le mariage de raison du musée d'art et du Web, Hermès $\mathrm{n}^{\circ} 61,2011$.

Tobelem, J-M. L'influence des nouvelles techniques sur le management des musées. Berlin, 2004, 16 p. www.archimuse.com/publishing/ichim04/0766_ Tobelem.pdf
Topalian, R. et Le Marec, J. Visite + : innover dans l'interactivité, la Lettre de l'OCIM, $\mathrm{n}^{\circ} 118,2008$. http://ocim.revues.org/342

Vidal, G. Interactivité et médiation dans l'usage des multimédias de musées, Communication et langages, $\mathrm{n}^{\circ} 137,2003$.

Leleu, N. (dir.) Musée 2.0. Centre Pompidou en partenariat avec le Centre Culturel Canadien à Paris, $1^{\text {er }}$ avril 2008. www.centrepompidou.fr/ Pompidou/Manifs.nsf/0/307C1B86BE7A06A6C12573AA0037BF62?Op enDocument\&session $\mathrm{M}=2.10 \& \mathrm{~L}=1$ 